\title{
Mental Hoop Diaries: Emotional Memories of a College Basketball Game in Rival Fans
}

\author{
Anne Botzung, David C. Rubin, Amanda Miles, Roberto Cabeza, and Kevin S. LaBar \\ Department of Psychology \& Neuroscience, Duke University, Durham, North Carolina 27708
}

The rivalry between the men's basketball teams of Duke University and the University of North Carolina-Chapel Hill (UNC) is one of the most storied traditions in college sports. A subculture of students at each university form social bonds with fellow fans, develop expertise in college basketball rules, team statistics, and individual players, and self-identify as a member of a fan group. The present study capitalized on the high personal investment of these fans and the strong affective tenor of a Duke-UNC basketball game to examine the neural correlates of emotional memory retrieval for a complex sporting event. Male fans watched a competitive, archived game in a social setting. During a subsequent functional magnetic resonance imaging session, participants viewed video clips depicting individual plays of the game that ended with the ball being released toward the basket. For each play, participants recalled whether or not the shot went into the basket. Hemodynamic signal changes time locked to correct memory decisions were analyzed as a function of emotional intensity and valence, according to the fan's perspective. Results showed intensity-modulated retrieval activity in midline cortical structures, sensorimotor cortex, the striatum, and the medial temporal lobe, including the amygdala. Positively valent memories specifically recruited processing in dorsal frontoparietal regions, and additional activity in the insula and medial temporal lobe for positively valent shots recalled with high confidence. This novel paradigm reveals how brain regions implicated in emotion, memory retrieval, visuomotor imagery, and social cognition contribute to the recollection of specific plays in the mind of a sports fan.

\section{Introduction}

A fundamental challenge in emotional memory research is to determine how the affective properties of complex, socially relevant events alter memory processes in the brain. Most neuroimaging studies have used simple stimuli that are not presented in social contexts and have little personal meaning to the individual participant. Although these studies have shown involvement of medial temporal lobe (MTL) structures during the retrieval of emotionally arousing stimuli (Buchanan, 2007), it is unclear whether these studies capture the brain systems that characterize emotional remembering in real-world settings. Indeed, when emotional intensity of autobiographical memories is specifically probed, there is mixed evidence for MTL sensitivity to this dimension of emotional experience (Maguire et al., 2003; Addis et al., 2004; Daselaar et al., 2008). Autobiographical memory also involves brain activity related to self-referential processing and sensorimotor imagery (e.g., Cabeza et al., 2004), although the sensitivity of these areas to emotional factors during retrieval is not well understood. Because autobiographical events are challenging to experimentally control for neuroimaging applications (Maguire, 2001), novel approaches are warranted to bridge a gap

Received May 27, 2009; revised Dec. 9, 2009; accepted Dec. 17, 2009.

This work was supported by United States National Institutes of Health Grants R01 AG023123 and P01 NS041328. We thank Jennifer Labrecque for her work in developing the basketball questionnaires and experimental paradigm, the Duke Department of Athletics for providing the video footage, and William Heath for video editing. We also thank Phil Kragel for assistance in $\mathrm{FMRI}$ analyses and Steven Green for help in creating the figures.

Correspondence should be addressed to Dr. Kevin S. LaBar, Duke University, Box 90999, Durham, NC 27708-0999. E-mail: klabar@duke.edu.

DOI:10.1523/JNEUROSCI.2481-09.2010

Copyright $\odot 2010$ the authors $\quad 0270-6474 / 10 / 302130-08 \$ 15.00 / 0$ in understanding how memories for emotional events in social settings are retrieved in the brain (Ochsner, 2008).

Here we develop a method to investigate the successful retrieval of highly emotional, self-relevant, realistic stimuli using a college basketball game paradigm in student fans from highranking rival teams. Fast passes, numerous shots, swings of emotion, and closeness of the score characterize a basketball game as an enjoyable social event and ensure that spectators maintain a high level of excitement. Fluxes in emotional valence are rapid and extreme, which permits the matching of intensity across positively and negatively valent events within the same participants over a short period of time. Plays that are emotionally positive for the fans of one team are emotionally negative for the fans of the other team, so the same exact event can be classified as either positive or negative according to the perspective of the fan. Potential confounds in events of opposing valence (i.e., differences in visual complexity) are thus completely controlled. Although dynamic event sequences are hard to parse into narrative episodes with unique emotions, individual plays of a basketball game provide segmental markers for memory assessment, each of which has its own emotional outcome. Because the consequence of a particular ball possession is not understood until the play is completely executed, the emotional impact is unknown until the memory for the end of the possession is recovered.

We predicted that emotional intensity ratings of specific plays would correlate with activity in the MTL, including the amygdala and hippocampus. Although valence effects were expected to be comparatively modest, we hypothesized that a positivity bias would be found in the left MTL and orbitofrontal cortex, consistent with the autobiographical memory literature (Markowitsch 
et al., 2003; Piefke et al., 2003). We were also interested in determining whether affective modulations would occur in structures that support self-referential processing and social cognition, including the temporoparietal junction and cortical midline structures, as well as neocortical areas involved in visuomotor imagery.

\section{Materials and Methods}

\section{Participants}

Twenty-three college basketball fans of high-ranking opposing teams, with no prior history of neurological or psychiatric impairment, participated in the study. All participants gave informed written consent before their inclusion in the study, which was approved by the Institutional Review Board at Duke University Medical Center. Twelve subjects were fans of the Duke University men's basketball team (mean age $=20.25$ years, $\mathrm{SD}=3.31$ ), and 11 subjects were fans of the rival team [University of North Carolina (UNC), mean age $=22.64$ years, $\mathrm{SD}=2.80$ ].

Individuals whose fan status is important for their self-identity experience intense emotions related to the team's performance and have greater physiological arousal during the competition than do individuals who are low in fan identification (Wann et al., 2003). Male sports fans exhibit neuroendocrine changes, such as testosterone surges following a team victory or testosterone drops following a team defeat, that parallel direct, one-on-one dominance contests (Bernhardt et al., 1998). Deeply committed fans are thus highly personally involved with the game despite experiencing it vicariously (Brown, 1986; Wann and Branscombe, 1993), which makes basketball memories in fans appropriate to study the brain systems that mediate retrieval of socially relevant, highly emotional events. For these reasons, participants were recruited through a set of questionnaires aimed at determining their level of fandom. The first two questionnaires involved self-ratings about the following: (1) their level of basketball fandom and their fan-related behavior with regards to their favorite men's college basketball team (adapted from Wann et al., 2003), (2) their own and others' perceptions of them as fans, as well as their feelings and behaviors associated with their fandom (adapted from Wann and Branscombe, 1993). Then, three questionnaires (see supplemental material, available at www.jneurosci.org) were created to determine their level of knowledge about basketball in general, National Collegiate Athletic Association men's basketball, and their favorite men's college basketball team (Duke or UNC). These questionnaires were constructed for the purpose of this study and piloted on 21 additional fans to select the most appropriate questions. The participants had to satisfy specific criteria to qualify for participation: they must have ranked themselves as having a high level of fandom, and maintained an overall average of $75 \%$ correct responses on all of the basketball knowledge questionnaires. Based on pilot data showing low-level memory performance for specific basket shots in female fans $(n=5$, mean correct recall $=60 \%$, chance $=50 \%$ ), only males were recruited to participate to the present study.

\section{Procedure and stimulus materials}

Learning phase. An archived competitive basketball game between Duke and UNC, played at UNC in 2001, was selected for the study. None of the participants were familiar with this particular game. The game footage was edited by eliminating commercials and portions with little action. Updates of the score were given for these missing sections. Participants watched the game in a comfortable laboratory setting three times over the course of a week before the scanning session, with the last encoding session always taking place $1 \mathrm{~d}$ before the scanning session. Based on pilot studies, this design ensured memory performance in a range of $70-80 \%$ accuracy. Most of the games were viewed in small groups of two to three individuals who were fans of the same team. The game was displayed on a large screen using an InFocus IN76 720p DLP Home Theater Projector.

Scanning phase. Participants were first given the instructions as well as a short practice session during which they were trained for the memory task to follow. In the scanner, they engaged in a cued recall task where they had to decide whether or not a particular shot released by a player went into the basket ( 1 = "in," 2 = "out"). Recall for each shot was contextually cued using 64 video clips originating from the viewed game. Each clip was $12 \mathrm{~s}$ in length and portrayed an action sequence leading up to a point where a player released a shot toward the basket. Shot clips were selected so that half $(n=32)$ would be emotionally positive according to one fan's perspective (good outcome for the team), whereas the other half would be emotionally negative (bad outcome for the team). Of course, the same shots that were bad for one team were good for the opponent team. The shots were also balanced for team possession [e.g., some of the emotionally positive shots were successfully made by the fan's own team ("makes") and some were shots missed by the opponent team ("misses")]. However, valence effects were not further subdivided according to team possession in the analyses due to the signal averaging requirements of functional magnetic resonance imaging (fMRI). All valence analyses were based on participants' own ratings of valence during scanning. Free throws and plays involving a change of team possession (i.e., steals) or fast breaks lasting $<12 \mathrm{~s}$ were excluded from the retrieval session. Across the rival fan perspectives, stimuli were thus matched both in terms of makes/misses and arousal levels but differed in valence. Importantly, this procedure eliminated any perceptual confounds across stimulus valence categories, since the same shots were used in all comparisons (i.e., the negative "stimulus" for the Duke fan also served as the positive "stimulus" for the UNC fan). The clips were shown in the game order. Presentation of each clip was followed by a $6 \mathrm{~s}$ fixation during which they were asked to recall whether the shot went in the basket ("yes" response) or not ("no" response), as indicated by a button press (see Fig. 1). The memory decision was followed by a memory confidence scale that appeared for $4.5 \mathrm{~s}$ (from $1=$ "very low" to $8=$ "very high"). Then an emotional rating scale appeared for $4.5 \mathrm{~s}$, which instructed the participants to rate each clip on a combined eight-point scale for valence and intensity (from -1 to -4 for negative events; or from 1 to 4 for positive events). The emotional rating was followed by short periods of fixation that were of jittered duration (mean duration $=3 \mathrm{~s}$, range $=2$ to $5 \mathrm{~s}$ ). The scanning session was divided into eight runs of eight trials each. Immediately before scanning, participants completed a short practice session to familiarize themselves with the memory task and response buttons.

\section{fMRI methods}

Scanning. Images were collected using a 4T GE scanner. Video clips were presented using liquid crystal display goggles (Resonance Technology), and sound was diffused through audio headphones. Behavioral responses were recorded using a Current Design eight-button serial response device. Cogent 2000 (UCL Institute of Cognitive Neuroscience) was used for the presentation and timing of stimuli. Scanner noise was reduced with earplugs, and head motion was minimized using foam pads and a headband. Anatomical scanning started with a T2-weighted sagittal localizer series. The anterior commissure and posterior commissure were identified in the midsagittal slice, and 34 contiguous oblique slices were prescribed parallel to the AC-PC plane. High-resolution T1-weighted structural images were collected first, with a $12.3 \mathrm{~ms}$ repetition time (TR), a $5.4 \mathrm{~ms}$ echo time (TE), a $24 \mathrm{~cm}$ field of view (FOV), a $256^{2}$ matrix, 68 slices, and a slice thickness of $1.9 \mathrm{~mm}$. Functional images were subsequently acquired using an inverse spiral sequence with a $1.5 \mathrm{~s}$ TR, a $31 \mathrm{~ms}$ TE, a $24 \mathrm{~cm}$ FOV, a $64^{2}$ matrix, and a $60^{\circ}$ flip angle. This sequence has been shown to reduce susceptibility artifact in the MTL (Wang et al., 2005). Thirty-four contiguous slices were acquired with the same slice prescription as the anatomical images. Slice thickness was $3.8 \mathrm{~mm}$, resulting in $3.75 \times 3.75 \times 3.8 \mathrm{~mm}$ isotropic voxels.

fMRI analyses. Preprocessing and statistical analyses were conducted using SPM5 software and implemented in MATLAB (Wellcome Department of Cognitive Neurology). Functional images were slice-timing corrected, realigned to correct for motion artifacts, spatially normalized to the Montreal Neurological Institute template, and then spatially smoothed using an $8 \mathrm{~mm}$ full-width at half-maximum isotropic Gaussian kernel.

Evoked hemodynamic responses time locked to the onset of the yes/no memory decision were modeled with a canonical hemodynamic response function. Hemodynamic activity related to the prior video clip presentation (recall cue) was modeled with a boxcar function, and the ratings were modeled as a variable of no interest. Although all trials were 
modeled, only correct memory trials (hits) were included in regressors of interest. The intensity ratings were considered in absolute values, and to homogenize the number of trials per intensity ranking, they were rescaled as follows: low $=1$, medium $=2$, high $=3-4$. The confidence ratings were rescaled from $1-8$ to 1-6 (with the first level including bins $1-3$ to homogenize ranking density). The valence ratings were grouped categorically as negative or positive. A parametric approach was first used to identify unique variance associated with intensity over fine-grained increments along the range of its scale. Intensity and confidence ratings were modeled linearly as two separate regressors. Confidence was orthogonalized with respect to intensity to derive unique variance estimates from intensity ratings. Individual contrast images of the parametric effect of intensity were then entered into a random-effects model using a one-sample $t$ test. Time courses were obtained using a finite impulse response (FIR) function model calculated for each variable level (i.e., high, medium, low).

Complementary categorical analyses were then conducted. Statistical parametric maps were generated for intensity (correct highly intense vs correct low intense events) and valence (correct positive events vs correct negative events), for each subject, in the context of the general linear model. This analysis also considered only correct memory trials (hits). The two sets of contrast images were subjected to random-effects analyses. Finally, to understand how memory confidence interacted with valence, a parametric analysis was performed using confidence ratings as a covariate of interest (modeled linearly). To take into account the fact that positive events were rated as more intense, intensity was modeled as a covariate of noninterest. The parametric analysis of confidence (orthogonalized to intensity) was conducted separately for positive and negative events at the first level, and the resulting contrast images were compared at the second level in a random-effects model using a paired $t$ test comparison.

For all these analyses, the significance threshold was set at $p=0.001$, with a minimum extent threshold of 10 contiguously activated voxels. Small volume correction (SVC) for multiple comparisons was used in a priori volumes of interest (amygdala and hippocampus) according to statistical procedures described by Worsley et al. (1996). Based on our predictions, separate masks for the SVC analyses were made for the intensity and valence comparisons. Anatomic masks were created using the Wake Forest University PickAtlas toolbox (Maldjian et al., 2003). The union of the anatomical regions selected from the aal atlas (TzourioMazoyer et al., 2002) and voxels significantly active for all correct responses were used to create all masks. This procedure ensured that the masks were anatomically precise and statistically independent of the contrasts to be tested. For examining the modulation of intensity, separate bilateral anatomical masks for the hippocampus and amygdala were used. For all valence comparisons, left-lateralized masks of the same regions were used, given that our hypotheses were lateralized for this comparison. The threshold for these regions was set a $p=0.05$, corrected for the familywise error rate.

\section{Results}

\section{Behavioral data}

The overall proportion of successfully recalled shots was 0.74 (see Table 1). Duke fans were faster than UNC fans to make responses in the memory decision task $\left(t_{(23)}=-3.03, p=0.006\right)$. However, there was no difference between Duke and UNC fans in terms of memory accuracy $\left(t_{(23)}=0.36, p=0.72\right)$, confidence $\left(t_{(23)}=\right.$ $-1.95, p=0.065)$, and intensity $\left(t_{(23)}=-0.82, p=0.42\right)$, nor in the reaction time measures of confidence $\left(t_{(23)}=-1.95, p=\right.$
Table 1. Mean (SD) behavioral data and reaction times (RTs, in milliseconds) for recall, confidence, and intensity judgments as a function of self-identified fan group

\begin{tabular}{llcc}
\hline Dependent measure & Duke fans & UNC fans & All subjects \\
\hline Recall & $75 \%(43)$ & $74 \%(44)$ & $74 \%(44)$ \\
Recall RT & $1383(856)^{*}$ & $1898(1126)$ & $1629(1026)$ \\
Confidence & $6.04(1.72)$ & $5.54(1.87)$ & $5.80(1.81)$ \\
Confidence RT & $863(683)$ & $1117(873)$ & $985(778)$ \\
Intensity & $1.98(0.97)$ & $1.85(0.86)$ & $1.91(0.91)$ \\
Intensity RT & $992(769)$ & $1260(935)$ & $1119(862)$ \\
\hline
\end{tabular}

${ }^{*} p<0.05$.

$0.065)$ and intensity $\left(t_{(23)}=-1.53, p=0.16\right)$. A significantly higher proportion of correct memories were positive than negative in valence, indicating a positivity bias in recall $\left(t_{(23)}=2.16\right.$, $p=0.042)$. Positive events were also rated with higher confidence $\left.t_{(23)}=3.42, p=0.002\right)$, and as being more intense than the negative ones $\left(t_{(23)}=3.67, p=0.001\right)$. This memory overconfidence for positive events confirms a prior behavioral study comparing memories for a Red Sox-Yankee game in fans of the opposing teams (Kensinger and Schacter, 2006). Although there were instances when fans made valence ratings during scanning that differed from the expected valence according to fan identity, participant's valence judgments for correctly recalled shots were on the average in the expected direction $88.9 \%$ of the time.

\section{fMRI data}

\section{Effects of emotional intensity}

Consistent with our predictions, emotional intensity modulated brain regions known to be involved in memory retrieval (prefrontal and temporal cortices), emotional processing [medial prefrontal cortex (mPFC), amygdala], the processing of selfrelevant and/or socially salient stimuli (anteromedial PFC and posterior cingulate, temporoparietal junction, and striatum), as well as regions that sustain visual (cuneus and precuneus) and motor (sensorimotor cortex, cerebellum, red nucleus) representations (Table 2 and Fig. 2). As indicated in Figure 2 (right), the time courses estimated through the FIR model support the para- 


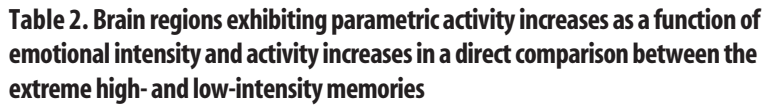
extreme high- and low-intensity memories

\begin{tabular}{|c|c|c|}
\hline Brain region & Z-score & Coordinates $(x, y, z)$ \\
\hline \multicolumn{3}{|l|}{ Parametric increases as a function of intensity } \\
\hline \multicolumn{3}{|l|}{ Prefrontal cortex } \\
\hline L inferior/mid-lateral PFC (BA 6/8/47) & 4.15 & $-41,30,38$ \\
\hline Medial dorsal PFC/anterior cingulate (BA 8/10/24) & 3.99 & $-19,64,19$ \\
\hline L inferior PFC (BA 47) & 3.55 & $-26,23,-19$ \\
\hline R mid-lateral PFC (BA 8/9) & 3.31 & $53,8,38$ \\
\hline \multicolumn{3}{|l|}{ Striatum and brainstem } \\
\hline L globus pallidus & 4.49 & $-23,-8,0$ \\
\hline R putamen/thalamus & 4.03 & $19,8,-8$ \\
\hline Bilateral red nucleus & 3.81 & $-8,-23,-4$ \\
\hline \multicolumn{3}{|l|}{ Posterior regions } \\
\hline L temporoparietal junction (BA 39/40) & 4.17 & $-45,-53,38$ \\
\hline L posterior cingulate (BA 30) & 3.58 & $-30,-71,11$ \\
\hline L precuneus (BA 7) & 3.25 & $-8,-75,56$ \\
\hline R cuneus (BA 18) & 3.05 & $11,-101,0$ \\
\hline L precuneus (BA 19) & 2.95 & $-30,-68,34$ \\
\hline \multicolumn{3}{|l|}{ Temporal lobes } \\
\hline L superior/middle temporal gyrus (BA 38/21) & 3.6 & $-45,4,-41$ \\
\hline R superior temporal gyrus (BA 41) & 3.27 & $45,-30,15$ \\
\hline R inferior temporal gyrus (BA 20) & 3.9 & $34,-8,-41$ \\
\hline R hippocampus & 3.63 & $19,-4,-15$ \\
\hline L parahippocampal gyrus (BA 36) & 2.84 & $-23,-30,-15$ \\
\hline L hippocampus & 2.67 & $-23,-26,-11$ \\
\hline R amygdala & 2.79 & $23,0,-11$ \\
\hline \multicolumn{3}{|l|}{ Sensorimotor regions } \\
\hline R sensorimotor cortex (BA 3/4/5) & 4.31 & $26,-23,64$ \\
\hline L cerebellum & 3.94 & $-23,-79,-30$ \\
\hline \multicolumn{3}{|l|}{ High intensity versus low intensity } \\
\hline \multicolumn{3}{|l|}{ Prefrontal cortex } \\
\hline L superior medial PFC (BA 6) & 4.04 & $-19,4,49$ \\
\hline Anteromedial PFC (BA 10) & 4.04 & $-8,49,0$ \\
\hline L inferior PFC/superior temporal gyrus (BA 47/38) & 3.78 & $-53,30,-8$ \\
\hline L middle PFC (BA 9) & 3.67 & $-45,30,38$ \\
\hline \multicolumn{3}{|l|}{ Basal ganglia and sensorimotor regions } \\
\hline R sensorimotor cortex (BA 3/4) & 5.22 & $30,-30,56$ \\
\hline R globus pallidus & 3.41 & $26,-19,0$ \\
\hline \multicolumn{3}{|l|}{ Posterior regions and temporal lobes } \\
\hline L temporoparietal junction (BA 39/40) & 4.16 & $-60,-49,41$ \\
\hline L precuneus/posterior cingulate gyrus (BA 31) & 3.78 & $-11,-53,34$ \\
\hline L inferior/middle temporal gyri (BA 37/21) & 3.72 & $-60,-56,-11$ \\
\hline L parahippocampal gyrus (BA 35) & 3.47 & $-23,-4,-26$ \\
\hline R amygdala/hippocampus & 3.47 & $23,0,-19$ \\
\hline
\end{tabular}

Only correct memory responses are included in the analyses. Coordinates are in Montreal Neurologic Institute stereotaxic space. BA, Brodmann area; L, left hemisphere; $R$, right hemisphere.

metric analysis. A follow-up planned contrast between high and low emotional intensity hits revealed that key regions of the memory, emotion, and social cognition circuitries were activated more for high-intensity trials, in accordance with the parametric model (see Table 2). The reverse comparison did not reveal any significant activity. An analysis of the interaction between emotional intensity and memory confidence yielded no significant effects.

The amygdala and hippocampal activations revealed by our primary intensity analyses were confirmed by a separate handdrawn anatomical region of interest (ROI) approach. A priori ROIs were drawn on the non-normalized individual T1-weighted structural scans in coronal section. Six subregions were identified: right and left amygdala, right and left anterior hippocampus, and right and left posterior hippocampus. Boundaries between subregions were identified using anatomical landmarks as described by Dolcos et al. (2004a). The general linear model and planned contrasts in the whole-brain analyses were replicated on the non-normalized functional data derived from the anatomical ROIs for each participant (averaged across all voxels in the ROI), and results were then averaged across participants for each ROI (see Table 3). These data were entered in an ANOVA to examine the effects of four factors on fMRI activity: intensity, valence, laterality, and region. This analysis revealed significant main effects of intensity $\left(F_{(2,22)}=9.129, p<0.001\right)$ and region $\left(F_{(2,22)}=3.080, p=0.047\right)$, but no effects of laterality $\left(F_{(1,22)}=0.174, p=0.677\right)$ or valence $\left(F_{(1,22)}=2.772, p=0.096\right)$. Follow-up tests showed that activity in these MTL ROIs was greater for high than for low $(p<0.001)$ and for medium than for low $(p=0.005)$ arousing shots, and that the posterior hippocampus showed greater signal change than the amygdala $(p=0.037)$. Therefore, this analysis confirmed the intensity modulation effects and differentiated activity levels within subregions of the MTL.

\section{Effects of emotional valence}

The analysis of the main effect of valence revealed a few regions specifically associated with the successful retrieval of positively valent shots relative to negatively valent shots. Positively valent memories yielded enhanced activity in a dorsal frontocinguloparietal network implicated in attentional control, as well as the right sensorimotor cortex (see Table 4 and Fig. 3). In contrast to the predictions, we did not observe valence-specific activity in the orbitofrontal cortex. To determine whether this was due to susceptibility artifacts, we quantified signal dropout in this region according to procedures detailed by Wang et al. (2005). Indeed, there were insufficient signal-to-noise estimates to reliably observe activation in the medial orbitofrontal cortex. No brain regions showed greater activity for negatively valent shots relative to positively valent ones. An analysis of the interaction between valence and memory confidence yielded additional activity in brain regions associated with memory retrieval processes (left hippocampus/parahippocampal cortex, left dorsolateral PFC), somatic representation (right insula), and multisensory integration (claustrum). These regions showed greater modulation by confidence for positive than negative valence, indicating a valence $\times$ memory interaction. No brain regions showed the opposite interaction (greater response to negative than positive shots as a function of memory confidence).

\section{Discussion}

The present study used fMRI in sports fans to reveal how memories are recalled for specific shots of a game, which contribute to oral traditions of sports history and a shared social experience among spectators. The Duke-UNC basketball rivalry in particular is a heralded sports tradition that is central to the personal identity of hardcore fans and a cornerstone of socialization in the local environment. Although prior research has examined behavioral memory performance in opposing fans of a baseball game (Kensinger and Schacter, 2006), it is unknown how event details of sporting competitions are recalled in the brain. Exploring the neural correlates of memories for specific plays in rival fans provides a unique research opportunity to characterize the play-byplay influence of emotional intensity and valence that combines the methodological advantages of autobiographical memory studies and laboratory studies using controlled unimodal cues.

\section{Recalling emotionally intense plays}

Although rival fans encode the actions of the game through opposing personal lenses, the findings indicate that successful memory retrieval is extensively modulated by emotional intensity 
across the fan perspectives. Emotionally intense plays tend to be those that break a tight score, elicit high drama (slam dunks), or come at key time points in the game (end of overtime). Only a handful of neuroimaging studies on autobiographical memory have used emotional intensity as a parametric regressor. Whereas Maguire and Frith (2003) and Addis et al. (2004) did not find any brain region modulated by intensity, Daselaar et al. (2008) reported multiple brain areas in which activity increased as a function of intensity ratings, including the amygdala, somatosensory cortices, and hippocampus. The results of the present study corroborate most of these latter effects using an entirely different methodological approach. The absence of modulation effects in the earlier studies might be related to the use of a prescan interview to collect the memories, which may reduce the role of emotional intensity during retrieval in the scanner (discussed in Daselaar et al., 2008). The retrieval of temporally disperse autobiographical events is also cognitively demanding, which can divert attentional resources from the emotional value of the memories (Phan et al., 2004). In this regard, the present design allows for less variability in terms of retrieval-related cognitive demand and encoding factors, perhaps rendering it more sensitive to detect influences of emotional intensity. Personal relevance also has been shown to enhance amygdala and precuneus responses during memory retrieval for the September 11, 2001 terrorist attacks in New York City. Sharot et al. (2007a) found that retrieval-related fMRI activity in these areas (as compared to a contemporaneous control event) was greater for individuals who experienced the attacks close to the World Trade Center site versus those who were located in Midtown Manhattan. As Rozin (2009) has noted, it is critical to demonstrate whether paradigmatic instances of behavior chosen for laboratory tasks extend to real-world events and are robust and generalizable.

The present study provides an important next step toward achieving this goal in the domain of emotional memory.

The modulation effect observed in both the hippocampus and the amygdala supports the importance of these two key structures in arousal-mediated memory retrieval (Dolcos et al., 2005; Buchanan, 2007). No valence-specific effect was found in the amygdala, which contributes to a growing body of evidence that challenges long-held notions regarding mnemonic specialization of the amygdala for negatively valenced stimuli (Hamann et al., 1999; Aggleton et al., 2000; Dolcos et al., 2004b, 2005). Although high emotional intensity is often readily elicited by negative sensory stimuli, the present study demonstrates how socially relevant contextual cues can be used to probe amyg-
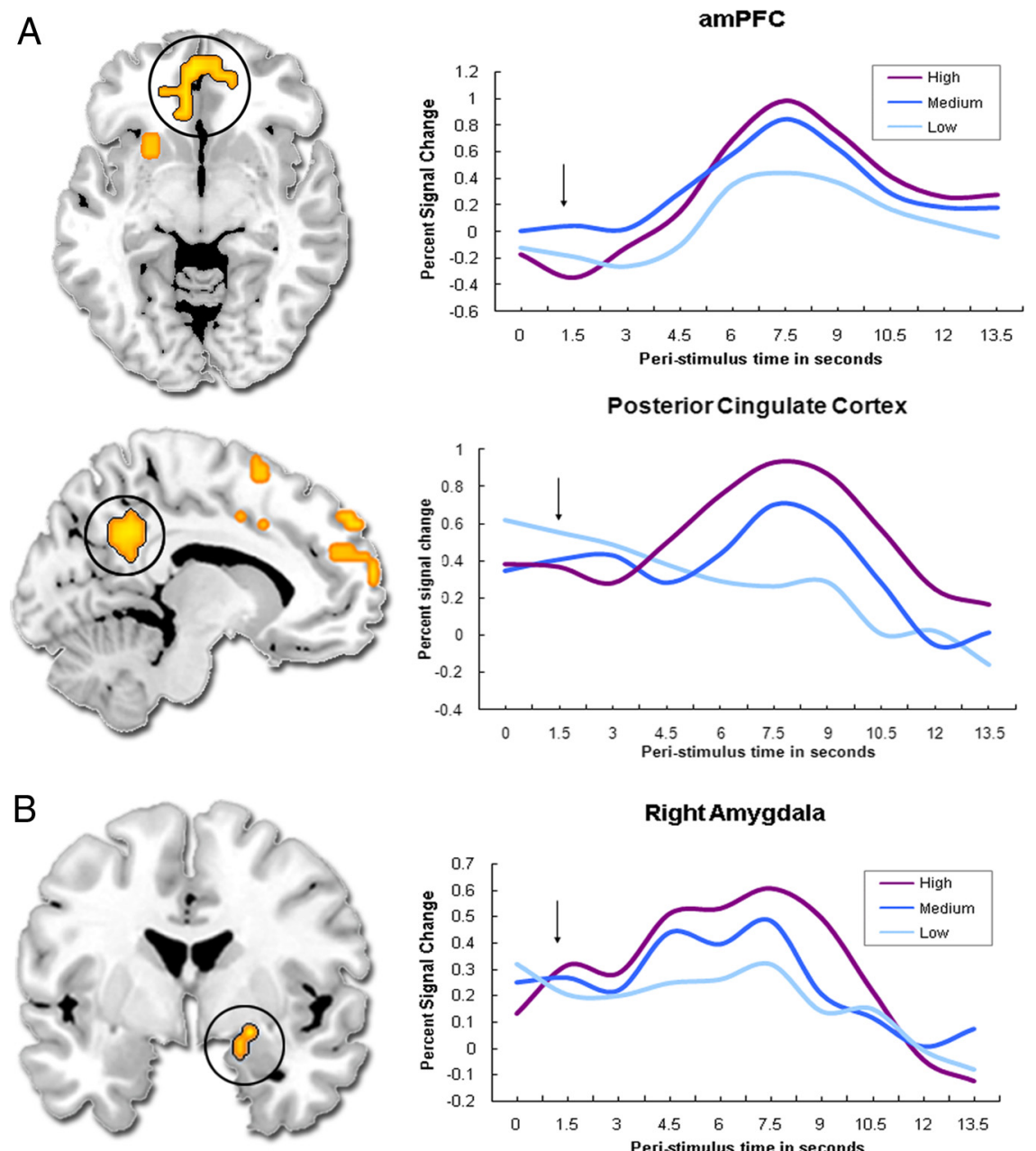

Right Amygdala

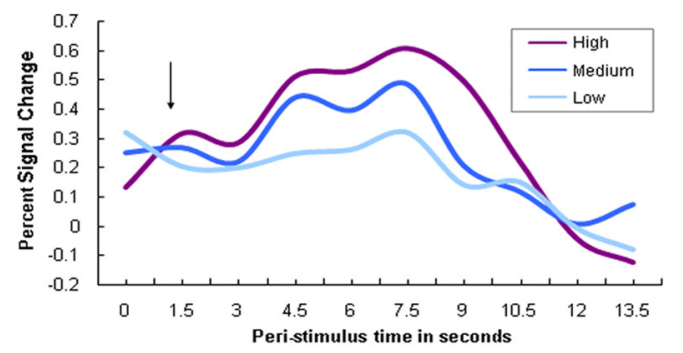

Right Hippocampus

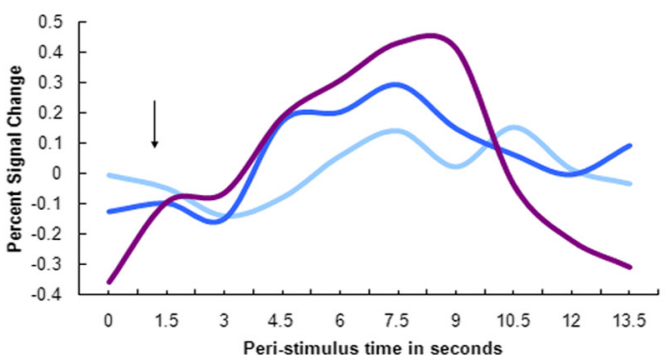

Figure 2. Memory activity parametrically modulated by increasing emotional intensity in cortical midline structures $(\boldsymbol{A})$ and in the medial temporal lobe $(\boldsymbol{B})$. The black arrow indicates when, on average, the memory decision was made. The time course plots indicate peak values within each region. amPFC, Anteromedial PFC.

dala sensitivity to intense, positively valenced memories (see also Greenberg et al., 2005).

In addition to the MTL, emotional intensity also enhanced activity in cortical midline structures such as the anteromedial $\mathrm{PFC}$ and posterior cingulate gyrus. Activity in these regions has been reported for a variety of social cognitive tasks, including self-judgments of personality traits (Craik et al., 1999; Kelley et al., 2002; D'Argembeau et al., 2005), past recollection and future projection (Addis and Schacter, 2008; Botzung et al., 2008a,b), and mentalizing [for review, see Buckner and Carroll (2007), Adolphs (2008), and Van Overwalle (2009)]. Our results contribute to this literature by implicating a tight coupling between the level of emotional intensity during recollection and putative so- 
Table 3. Mean (SE) fMRI signal values associated with successful retrieval of high-, medium-, and low-intensity trials, calculated in each medial temporal lobe ROI (averaged across all voxels) in the context of the general linear model

\begin{tabular}{llll}
\hline & High & Medium & Low \\
\hline Amygdala & & & \\
$\quad$ Right & $2.43(0.50)$ & $1.91(0.44)$ & $0.99(0.41)$ \\
Left & $2.26(0.57)$ & $1.51(0.36)$ & $0.87(0.44)$ \\
$\quad$ Average & $2.35(0.54)$ & $1.71(0.40)$ & $0.93(0.43)$ \\
$\begin{array}{l}\text { Hippocampus } \\
\text { Anterior }\end{array}$ & & & \\
$\quad$ Right & $2.57(0.50)$ & $2.25(0.39)$ & $1.87(0.40)$ \\
$\quad$ Left & $2.79(0.49)$ & $2.63(0.40)$ & $2.08(0.47)$ \\
$\quad$ Average & $2.68(0.50)$ & $2.44(0.40)$ & $1.98(0.44)$ \\
Posterior & & & \\
$\quad$ Right & $2.78(0.71)$ & $2.04(0.52)$ & $1.44(0.52)$ \\
$\quad$ Left & $3.09(0.48)$ & $2.54(0.38)$ & $2.57(0.45)$ \\
Average & $2.94(0.6)$ & $2.29(0.45)$ & $2.01(0.49)$ \\
\hline
\end{tabular}

Table 4. Brain regions exhibiting a main effect of valence (increased activity for positive relative to negative events) and a valence $\times$ memory interaction (increased activity for positive relative to negative events only in the context of high memory confidence)

\begin{tabular}{llc}
\hline Brain region & Z-score & Coordinates $(x, y, z)$ \\
\hline Positive $>$ negative valence & & \\
$\quad$ R sensorimotor cortex (BA 3/6) & 3.81 & $26,-4,68$ \\
L superior PFC (BA 9) & 3.46 & $-38,45,30$ \\
$\quad$ R superior parietal lobule (BA 40) & 3.54 & $64,-23,15$ \\
$\quad$ R inferior parietal lobule (BA 40) & 3.33 & $45,-49,60$ \\
$\quad$ ingulate gyrus (BA 24) & 3.29 & $0,-4,38$ \\
Parametric effect of increasing confidence on & & \\
$\quad$ positive $>$ negative valence & & \\
R insula/superior temporal gyrus (BA 13/22) & 3.4 & $53,11,-4$ \\
L parahippocampal gyrus (BA 35)/hippocampus & 3.36 & $-19,-23,-19$ \\
L claustrum & 3.13 & $-26,-4,19$ \\
L superior temporal gyrus (BA 22) & 3.04 & $-53,11,0$ \\
L hippocampus & 2.87 & $-34,-34,-8$ \\
L dorsolateral PFC (BA 10/46) & 2.81 & $-34,41,19$ \\
\hline
\end{tabular}

Only correct memory responses are included in the analyses. Coordinates are in Montreal Neurologic Institute stereotaxic space. BA, Brodmann area; $L$, left hemisphere; $R$, right hemisphere.
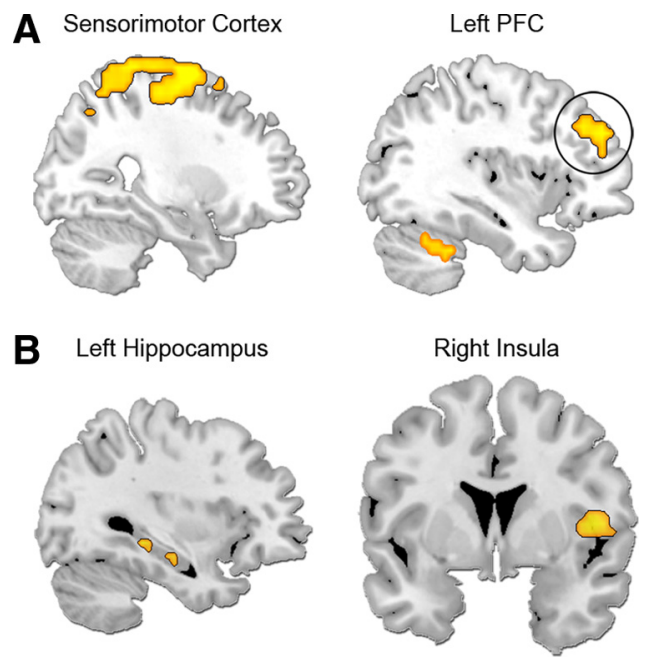

Figure 3. Regions showing increased activity for positive relative to negative event memories $(\boldsymbol{A})$ and an interaction between valence and memory confidence $(\boldsymbol{B})$. Positively valent memories specifically elicited activity in the sensorimotor cortex and left lateral PFC $(\boldsymbol{A})$. The difference between positive and negative memories was magnified in additional regions, including the insula and hippocampus, when memory confidence was high $(\boldsymbol{B})$. cial cognition-related activity in these and related regions, such as the temporoparietal junction and superior temporal gyrus. In the context of the present study design, emotionally intense plays may have recruited these regions due to perspective taking/mentalizing of player thoughts or feelings or simulating the interpersonal dynamics of the shot (assessing the relative positioning and actions of players nearby). It is also very likely that emotionally intense plays recruited self-referential processing ("how did I feel about this shot") or recollections of the social setting (reactions of the crowd or other research participants). We note that most participants had personally attended other games in the UNC arena, so they could also draw upon their own first-hand experience with the setting while making memory judgments (unlike recall of movie clips that take place in unfamiliar settings). The posterior cingulate is hypothesized to support the integration of self-relatedness and memory, whereas the temporoparietal junction supports inferences of immediate action goals and intentions (Northoff and Bermpohl, 2004). Information processing about unfamiliar others selectively engages the dorsal part of the $\mathrm{mPFC}$, whereas the ventral part is implicated when processing core experiences of the self and familiar others (Van Overwalle, 2009). Our data are consistent with this claim, since our participants had to recall information involving both their team (familiar other) and the opponent team (unfamiliar other). Although the specific contributions of these structures is currently speculative, the sporting event paradigm provides a rich setting to probe the relationship between brain areas and selective social cognitive processes in future studies.

Cortical visuomotor and striatal regions are also noteworthy for their sensitivity to emotional intensity. The cuneus/precuneus has long been implicated in visual imagery during memory tasks, and emotional intensity selectively enhances the sensory vividness of autobiographical memories (Fletcher et al., 1995; Rubin et al., 2003; Talarico et al., 2004). Emotional arousal may also enhance motor imagery and motor memory processes in the cerebellum, red nucleus, and somatosensory/motor cortices in the context of the recollection of specific play actions in the game [see also Nyberg et al. (2001) and Rubin (2005)]. The striatum may be involved because basketball shooting is a skilled motor action, and memory accuracy within the context of emotional arousal may require greater attention to procedural aspects of the unfolding action sequence, given that nuances in wrist release, balance, and other motor factors influence shooting precision. Alternatively, its role may relate to emotional modulation of cue-outcome predictions (Thomas and LaBar, 2008), especially since the contextual retrieval cues and outcomes are highly redundant in the task. In either case, the present results extend the known role of the striatum in memory-guided decision making for personally salient events.

\section{Recalling positively versus negatively valent plays}

There was both behavioral and brain evidence for a positivity bias in memory recall. Positively valent shots (shots made by the fan's team or missed by the rival team) were rated as more emotionally intense than negatively valent shots, and they were also recalled with greater accuracy and with more confidence. Interestingly, this pattern is opposite to that typically found using basic sensory stimuli, in which negative stimuli more readily elicit highly intense affect. After controlling for differences in emotional intensity in the regression model, the positivity bias was associated with recruitment of dorsal frontocinguloparietal areas that may reflect greater spread of attentional allocation, in support of the broaden-and-build model of positive emotions (Fredrickson, 
2001). Previous studies have also shown greater rostral cingulate activity for individuals who exhibit optimism bias in imagining future events (Sharot et al., 2007b), and greater dorsal frontocinguloparietal activity in a subsequent memory paradigm for positive stimuli relative to negative and neutral stimuli (Mickley and Kensinger, 2008). Differential allocation of attention could, in turn, recruit enhanced sensorimotor cortical representations, which were also selectively activated by positive affect, as well as the left superior PFC (area BA 9), which has been implicated in sustaining retrieval processes (Cabeza et al., 2003; Botzung et al., 2008a). Other brain areas selectively contributed to memory confidence within the context of positive (but not negative) events, including the right insula, claustrum, medial and lateral temporal lobe, and dorsolateral PFC. We postulate that the PFC and MTL activity might reflect a benefit on recollection processes associated with the recall of these positive social stimuli. The claustrum is hypothesized to facilitate the communication between sensory cortices belonging to different sensory modalities (Kayser et al., 2005), which may underlie strong multimodal representations during recollection for positive social events. The right insula serves as a convergence point for representations of bodily states with social and contextual information to provide a neural substrate of the conscious experience of our own emotions (Craig, 2002) as well as the empathic feeling of others' emotions (Adolphs, 2008). These processes may be amplified during pleasant social memories recalled with high confidence. In general, the additional recruitment of brain regions associated with positive memories is consistent with the autobiographical memory literature (Markowitsch et al., 2003; Piefke et al., 2003), although we could not identify significant positivity effects in the orbitofrontal cortex due to signal dropout.

\section{Conclusions}

In conclusion, a novel experimental paradigm was developed to assess the neural correlates of emotional memory in an enjoyable sporting event setting for fans characterized by high expertise in the game and personal identification with players of a particular team. Not only do the results replicate prior findings of arousalmediated memory functions of the amygdala and hippocampus, but they also implicate an extended social cognitive and sensorimotor network that is not typically associated with emotional recall using simple sensory stimuli. Unlike typical autobiographical memory studies, this paradigm provides a verifiable context for emotional intensity and valence and a compressed encoding time scale. The paradigm also provides an ethical way to explore memory for emotions that are experienced as intensely negative and positive in research participants. Despite strong feelings of opposition to the rival team, this study revealed an emotioninduced resonance in brain activity across rival fans while reflecting on emotionally intense plays of a competitive game. Future studies could compare recall as a function of encoding setting (televised viewing vs actual game attendance) to determine whether the results extend to fans who have first-hand experiences of the game in its physical context. Finally, the results should be extended to female fans and female team competitions. Although memory performance for shot outcomes was not sufficiently high in our pilot study of female fans to enable inclusion in the imaging analysis, memory for other aspects of the game may be more amenable for investigation, such as remembering personal aspects of the experience (Kensinger and Schacter, 2006). In sum, this study offers an innovative direction for integrating controlled, realistic, and complex social experiences into the neuroimaging field, and constitutes a bridge between laboratory-based and autobiographical studies of emotional remembering.

\section{References}

Addis DR, Schacter DL (2008) Constructive episodic simulation: temporal distance and detail of past and future events modulate hippocampal engagement. Hippocampus 18:227-237.

Addis DR, Moscovitch M, Crawley AP, McAndrews MP (2004) Recollective qualities modulate hippocampal activation during autobiographical memory retrieval. Hippocampus 14:752-762.

Adolphs R (2008) Fear, faces, and the human amygdala. Curr Opin Neurobiol 18:166-172.

Aggleton JP, Vann SD, Oswald CJP, Good M (2000) Identifying cortical inputs to the rat hippocampus that subserve allocentric spatial processes: a simple problem with a complex answer. Hippocampus 10:466-474.

Bernhardt PC, Dabbs JM Jr, Fielden JA, Lutter CD (1998) Testosterone changes during vicarious experiences of winning and losing among fans at sporting events. Physiol Behav 65:59-62.

Botzung A, Denkova E, Ciuciu P, Scheiber C, Manning L (2008a) The neural bases of the constructive nature of autobiographical memories studied with a self-paced fMRI design. Memory 16:351-363.

Botzung A, Denkova E, Manning L (2008b) Experiencing past and future personal events: functional neuroimaging evidence on the neural bases of mental time travel. Brain and Cognition 66:202-212.

Brown R (1986) Social psychology, Ed 2, pp 551-556. New York: The Free Press.

Buchanan TW (2007) Retrieval of emotional memories. Psychol Bull 133:761-779.

Buckner RL, Carroll DC (2007) Self-projection and the brain. Trends Cogn Sci 11:49-57.

Cabeza R, Locantore JK, Anderson ND (2003) Lateralization of prefrontal activity during episodic memory retrieval: evidence for the productionmonitoring hypothesis. J Cogn Neurosci 15:249-259.

Cabeza R, Prince SE, Daselaar SM, Greenberg DL, Budde M, Dolcos F, LaBar KS, Rubin DC (2004) Brain activity during episodic retrieval of autobiographical and laboratory events: an fMRI study using a novel photo paradigm. J Cogn Neurosci 16:1583-1594.

Craig AD (2002) How do you feel? Interoception: the sense of the physiological condition of the body. Nat Rev Neurosci 3:655-666.

Craik FIM, Moroz TM, Moscovitch M, Stuss DT, Winocur G, Tulving E, Kapur S (1999) In search of the self: a positron emission tomography study. Psychol Sci 10:26-34.

D’Argembeau A, Collette F, Van der Linden M, Laureys S, Del Fiore G, Degueldre C, Luxen A, Salmon E (2005) Self-referential reflective activity and its relationship with rest: a PET study. Neuroimage 25:616-624.

Daselaar SM, Rice HJ, Greenberg DL, Cabeza R, LaBar KS, Rubin DC (2008) The spatiotemporal dynamics of autobiographical memory: neural correlates of recall, emotional intensity, and reliving. Cereb Cortex 18:217-229.

Dolcos F, LaBar KS, Cabeza R (2004a) Interaction between the amygdala and the medial temporal lobe memory system predicts better memory for emotional events. Neuron 42:955-963.

Dolcos F, LaBar KS, Cabeza R (2004b) Dissociable effects of arousal and valence on prefrontal activity indexing emotional evaluation and subsequent memory: an event-related fMRI study. Neuroimage 23:64-74.

Dolcos F, LaBar KS, Cabeza R (2005) Remembering one year later: role of the amygdala and the medial temporal lobe memory system in retrieving emotional memories. Proc Natl Acad Sci U S A 102:2626-2631.

Fletcher PC, Frith CD, Baker SC, Shallice T, Frackowiak RSJ, Dolan RJ (1995) The mind's eye-precuneus activation in memory related imagery. Neuroimage 2:195-200.

Fredrickson BL (2001) The role of positive emotions in positive psychology. The broaden-and-build theory of positive emotions. Am Psychol $56: 218-226$.

Greenberg DL, Rice HJ, Cooper JJ, Cabeza R, Rubin DC, LaBar KS (2005) Co-activation of the amygdala, hippocampus and inferior frontal gyrus during autobiographical memory retrieval. Neuropsychologia 43:659-674.

Hamann SB, Ely TD, Grafton ST, Kilts CD (1999) Amygdala activity related to enhanced memory for pleasant and aversive stimuli. Nat Neurosci 2:289-293. 
Kayser C, Petkov CI, Augath M, Logothetis NK (2005) Integration of touch and sound in auditory cortex. Neuron 48:373-384.

Kelley WM, Macrae CN, Wyland CL, Caglar S, Inati S, Heatherton TF (2002) Finding the self? An event-related study. J Cogn Neurosci 14:785-794.

Kensinger EA, Schacter DL (2006) When the Red Sox shocked the Yankees: comparing negative and positive memories. Psychon Bull Rev 13:757-763.

Maguire EA (2001) Neuroimaging studies of autobiographical event memory. Philos Trans R Soc Lond B Biol Sci 356:1441-1451.

Maguire EA, Frith CD (2003) Lateral asymmetry in the hippocampal response to the remoteness of autobiographical memories. J Neurosci 23:5302-5307.

Maldjian JA, Laurienti PJ, Kraft RA, Burdette JH (2003) An automated method for neuroanatomic and cytoarchitectonic atlas-based interrogation of fMRI data sets. Neuroimage 19:1233-1239.

Markowitsch HJ, Vandekerckhove MMP, Lanfermann H, Russ MO (2003) Engagement of lateral and medial prefrontal areas in the ecphory of sad and happy autobiographical memories. Cortex 39:643-665.

Mickley KR, Kensinger EA (2008) Emotional valence influences the neural correlates associated with remembering and knowing. Cogn Affect Behav Neurosci 8:143-152.

Northoff G, Bermpohl F (2004) Cortical midline structures and the self. Trends Cogn Sci 8:102-107.

Nyberg L, Petersson K-M, Nilsson L-G, Sandblom J, Åberg C, Ingvar M (2001) Reactivation of motor brain areas during explicit memory for actions. Neuroimage 14:521-528.

Ochsner KN (2008) The social-emotional processing stream: five core constructs and their translational potential for schizophrenia and beyond. Biol Psychiat 64:48-61.

Phan KL, Wager TD, Taylor SF, Liberzon I (2004) Functional neuroimaging studies of human emotions. CNS Spectr 9:258-266.

Piefke M, Weiss PH, Zilles K, Markowitsch HJ, Fink GR (2003) Differential remoteness and emotional tone modulate the neural correlates of autobiographical memory. Brain 126:650-668
Rozin P (2009) What kind of empirical research should we publish, fund, and reward? A different perspective. Perspect Psychol Sci 4:435-439.

Rubin DC (2005) A basic-systems approach to autobiographical memory. Curr Dir Psychol Sci 14:79-83.

Rubin DC, Schrauf RW, Greenberg DL (2003) Belief and recollection of autobiographical memories. Mem Cognit 31:887-901.

Sharot T, Martorella EA, Delgado MR, Phelps EA (2007a) How personal experience modulates the neural circuitry of memories of September 11. Proc Natl Acad Sci U S A 104:389-394.

Sharot T, Riccardi AM, Raio CM, Phelps EA (2007b) Neural mechanisms mediating optimism bias. Nature 450:102-105.

Talarico J, LaBar KS, Rubin D (2004) Emotional intensity predicts autobiographical memory experience. Mem Cognit 32:1118-1132.

Thomas LA, LaBar KS (2008) Fear relevancy, strategy use, and probabilistic learning of cue-outcome associations. Learn Mem 15:777-784.

Tzourio-Mazoyer N, Landeau B, Papathanassiou D, Crivello F, Etard O, Delcroix N, Mazoyer B, Joliot M (2002) Automated anatomical labeling of activations in SPM using a macroscopic anatomical parcellation of the MNI MRI single-subject brain. Neuroimage 15:273-289.

Van Overwalle F (2009) Social cognition and the brain: a meta-analysis. Hum Brain Mapp 30:829-858.

Wang L, McCarthy G, Song AW, LaBar KS (2005) Amygdala activation to sad pictures during high-field (4 tesla) functional magnetic resonance imaging. Emotion 5:12-22.

Wann DL, Branscombe NR (1993) Sports fans: measuring degree of identification with their team. Int J Sport Psychol 24:1-17.

Wann DL, Friedman K, McHale M, Jaffe A (2003) The Norelco Sport Fanatics Survey: examining behaviors of sport fans. Psychol Rep 92:930936.

Worsley KJ, Marrett S, Neelin P, Vandal AC, Friston KJ, Evans AC (1996) A unified statistical approach for determining significant signals in images of cerebral activation. Hum Brain Mapp 4:58-73. 